PROCEEDINGS OF THE

AMERICAN MATHEMATICAL SOCIETY

Volume 137, Number 12, December 2009, Pages 4287-4294

S 0002-9939(09)09985-7

Article electronically published on July 16, 2009

\title{
EMBEDDING 3-MANIFOLDS WITH CIRCLE ACTIONS
}

\author{
J. A. HILLMAN
}

(Communicated by Daniel Ruberman)

\begin{abstract}
Constraints on the Seifert invariants of orientable 3-manifolds $M$ which admit fixed-point free $S^{1}$-actions and embed in $\mathbb{R}^{4}$ are given. In particular, the generalized Euler invariant of the orbit fibration is determined up to sign by the base orbifold $B$ unless $H_{1}(M ; \mathbb{Z})$ is torsion free, in which case it can take at most one nonzero value (up to sign). An $\mathbb{H}^{2} \times \mathbb{E}^{1}$-manifold $M$ with base orbifold $B=S^{2}\left(\alpha_{1}, \ldots, \alpha_{r}\right)$ where all cone point orders are odd embeds in $\mathbb{R}^{4}$ if and only if its Seifert data $S$ is skew-symmetric.
\end{abstract}

The question of which closed 3-manifolds $M$ (other than homology spheres) embed in $\mathbb{R}^{4}$ has received surprisingly little attention. (The relevant papers known to us are $[2,3,5-11]$.) In particular, it is not yet known which Seifert fibred 3manifolds embed, although in many other respects this is a well-understood class of spaces, with natural parametrizations in terms of Seifert data. It was shown earlier that if $M$ embeds in $\mathbb{R}^{4}$, then it must be orientable and the torsion subgroup $T(M)$ of $H_{1}(M ; \mathbb{Z})$ must be a direct double: $T(M) \cong U \oplus U$ for some finite abelian group $U$ [8]. Moreover, the linking pairing $\ell_{M}$ on $T(M)$ must be hyperbolic [13. Most of the known constructions give smooth embeddings, but these conditions must also hold if $M$ embeds as a TOP locally flat submanifold.

The first two sections establish our notation and summarize some basic facts about compact 3 -manifolds in $\mathbb{R}^{4}$. In $\S 3$ we shall observe that $T(M)$ being a direct double imposes strong constraints on the Seifert data of orientable 3-manifolds which admit fixed-point free $S^{1}$-actions and which embed in $\mathbb{R}^{4}$. The present simple argument does not work for orientable Seifert fibred 3-manifolds with nonorientable base orbifolds. These admit no compatible $S^{1}$-action. However in 3 we used the $Z / 2 Z$-index theorem to constrain the Euler invariants for such 3-manifolds. As a consequence we were able to settle there the question of embeddability for manifolds having one of the geometries $\mathbb{S}^{3}, \mathbb{E}^{3}, \mathbb{N} i l^{3}, \mathbb{S}^{2} \times \mathbb{E}^{1}$ or $\mathbb{S o l} l^{3}$.

When the Seifert data is "skew-symmetric" (i.e., is a set of complementary pairs) and all cone point orders are odd, the corresponding Seifert manifold embeds smoothly [3. Such a manifold has generalized Euler invariant $\varepsilon=0$ and so is geometric of type $\mathbb{E}^{3}, \mathbb{S}^{2} \times \mathbb{E}^{1}$ or $\mathbb{H}^{2} \times \mathbb{E}^{1}$. In $\S 4$ we use the $G$-index theorem again to obtain our main result, Theorem 4.4:

Received by the editors January 19, 2009, and, in revised form, April 2, 2009

2000 Mathematics Subject Classification. Primary 57N10; Secondary 57N13.

Key words and phrases. Embedding, Euler invariant, linking pairing, Seifert bundle.

This paper began as a 1998 University of Sydney Research Report, but the main result was obtained while the author was visiting the University of Durham as the Grey College Mathematics Fellow for Michaelmas Term of 2008 .

(C)2009 American Mathematical Society Reverts to public domain 28 years from publication 
Let $M$ be an orientable Seifert fibred 3-manifold $M$ whose base orbifold is a marked 2-sphere $B=S^{2}\left(\alpha_{1}, \ldots, \alpha_{r}\right)$, with all $\alpha_{i}$ odd and $\varepsilon=0$. Then $M$ embeds in $\mathbb{R}^{4}$ if and only if the Seifert data is skew-symmetric.

(There is a somewhat stronger result below, but we do not yet know whether skewsymmetry is necessary for embeddability when $g>0$.)

The final section gives some remarks on the cases not covered by our main result, namely when the base orbifold is nonorientable or when $\varepsilon \neq 0$.

\section{SEIFERT DATA AND BILINEAR PAIRINGS}

An orientable 3-manifold admits a fixed-point free $S^{1}$-action if and only if it is Seifert fibred over an orientable base orbifold. Let $T_{g}$ be the orientable surface of genus $g$, and let $M=M(g ; S)$ be the orientable Seifert fibred 3-manifold with base orbifold $B=T_{g}\left(\alpha_{1}, \ldots, \alpha_{r}\right)$ and Seifert data $S=\left\{\left(\alpha_{1}, \beta_{1}\right), \ldots,\left(\alpha_{r}, \beta_{r}\right)\right\}$, where $1<\alpha_{i}$ and $\left(\alpha_{i}, \beta_{i}\right)=1$, for all $1 \leq i \leq r$. (Our notation is based on that of [1]. In particular, we do not assume that $0<\beta_{i}<\alpha_{i}$.) If $r=1$, we allow also the possibility that $\alpha_{1}=1$. Let $\varepsilon_{S}=-\sum_{i=1}^{i=r}\left(\beta_{i} / \alpha_{i}\right)$ be the generalized Euler invariant

of the Seifert bundle, and let $\Pi=\prod_{i=1}^{i=r} \alpha_{i}$. (Replacing each $\beta_{i}$ by $\eta \beta_{i}+c_{i} \alpha_{i}$ where $\eta= \pm 1$ and $\sum c_{i}=0$ gives a homeomorphic manifold.) As $B$ is the connected sum of $T_{g}$ and $S^{2}\left(\alpha_{1}, \ldots, \alpha_{r}\right)$ we have $M=M(0 ; S) \#_{f} M(g ; \emptyset)=M(0 ; S) \#_{f}\left(T_{g} \times S^{1}\right)$, where $\#_{f}$ denotes fibre sum.

We shall say that the Seifert data $S=\left\{\left(\alpha_{1}, \beta_{1}\right), \ldots,\left(\alpha_{r}, \beta_{r}\right)\right\}$ is skew-symmetric if $r$ is even and if $\alpha_{2 i-1}=\alpha_{2 i}$ and $\beta_{2 i-1}=-\beta_{2 i}$ for $1 \leq i \leq r / 2$.

If $M$ is a closed oriented 3-manifold, Poincaré duality determines a linking pairing $\ell_{M}: T(M) \times T(M) \rightarrow \mathbb{Q} / \mathbb{Z}$. This is symmetric, bilinear, and nonsingular in the sense that the adjoint function $\widetilde{\ell_{M}}: m \mapsto \ell(-, m)$ defines an isomorphism from $T(M)$ to $\operatorname{Hom}(T(M), \mathbb{Q} / \mathbb{Z})$.

There are analogous pairings on covering spaces of $M$. In particular, if $\phi$ : $\pi_{1}(M) \rightarrow \mathbb{Z}$ is an epimorphism with associated covering space $M_{\phi}$, the homology modules $H_{1}\left(M_{\phi} ; R\right)$ are $R \Lambda$-modules, where $\Lambda=\mathbb{Z}[\mathbb{Z}]=\mathbb{Z}\left[t, t^{-1}\right]$ and $R \Lambda=R \otimes_{\mathbb{Z}} \Lambda$, for any coefficient ring $R$. Let $H_{i}(M ; \mathbb{Q} \Lambda)=H_{i}\left(M_{\phi} ; \mathbb{Q}\right)$ and $H_{i}(M ; \mathbb{Q}(t))=\mathbb{Q}(t) \otimes_{\Lambda}$ $H_{i}(M ; \mathbb{Q} \Lambda)$. There is a Blanchfield pairing $b_{\phi}$ on the $\mathbb{Q} \Lambda$-torsion submodule of $H_{1}(M ; \mathbb{Q} \Lambda)$ with values in $\mathbb{Q}(t) / \mathbb{Q} \Lambda$ which is nonsingular and hermitian with respect to the involution sending $t$ to $t^{-1}$. When $\phi$ corresponds to a fibre bundle projection from $M$ to $S^{1}$ with fibre $F$ being a closed surface, then $b_{\phi}$ is equivalent to the isometric structure given by the intersection pairing $I_{F}$ on $H_{1}(F ; \mathbb{Q})$ together with the isometric action of $Z$. (See Appendix A of [15].) Such a pairing is neutral if the underlying $\mathbb{Q} \Lambda$-torsion module has a submodule which is its own annihilator with respect to the pairing. (See Chapter 2 of [9].) Two such pairings are Wittequivalent if they become isomorphic after addition of suitable neutral pairings. The Witt group of isometric structures on finite dimensional $\mathbb{Q}$-vector spaces is the set $W(\mathbb{Q}(t), \mathbb{Q} \Lambda)$ of Witt equivalence classes of such pairings, with the addition induced by the direct sum of pairings. (See [16].)

\section{SOME BASIC OBSERVATIONS ON EMBEDDINGS}

A 3-manifold $M$ embeds in $\mathbb{R}^{4}$ if and only if it embeds in $S^{4}$. Since our arguments are largely homological it is more natural to assume that $M$ embeds in an homology 4-sphere $\Sigma$. The complement $\Sigma \backslash M$ has two components, and the closures $X$ and $Y$ of these components have boundary $M$. The Mayer-Vietoris sequence of the triple 
$(\Sigma, X, Y)$ gives $H_{i}(M ; R) \cong H_{i}(X ; R) \oplus H_{i}(Y ; R)$ for $i=1,2$ and $H_{i}(X ; R)=$ $H_{i}(Y ; R)=0$ for $i>2$, for any simple coefficients $R$. In particular, $\chi(X)+\chi(Y)=$ 2 , and $\chi(X) \equiv \chi(Y) \equiv 1+\beta_{1}(M ; \mathbb{Q}) \bmod (2)$.

Similar results apply for cohomology. Thus $H^{1}(M ; \mathbb{Z}) \cong H^{1}(X ; \mathbb{Z}) \oplus H^{1}(Y ; \mathbb{Z})$ has a basis consisting of epimorphisms which extend on one side or the other, while Alexander duality gives isomorphisms $H^{1}(X ; R) \cong H_{2}(Y ; R)$ and $H^{2}(X ; R) \cong$ $H_{1}(Y ; R)$.

Let $T_{X}$ and $T_{Y}$ be the kernels of the induced homomorphisms from $T(M)$ to $H_{1}(X ; \mathbb{Z})$ and $H_{1}(Y ; \mathbb{Z})$, respectively. Then $T_{M} \cong T_{X} \oplus T_{Y}$. It follows easily from Poincare duality that the restriction of $\ell_{M}$ to each of these summands is trivial, and so $\ell_{M}$ is hyperbolic [13. In particular, $T(X) \cong \operatorname{Hom}(T(Y), \mathbb{Q} / \mathbb{Z})$, which is non-canonically isomorphic to $T(Y)$, and so $T(M)$ is a direct double.

If a Seifert fibred 3-manifold $M$ embeds in $S^{4}$, then so does $M \#_{f}\left(T_{g} \times S^{1}\right)$, by Lemma 3.2 of 3 . For our main result we shall need to assume that $M=M(0 ; S)$. Thus it would be very convenient to have a converse to this stabilization result. (The analogous implication in the case of nonorientable base orbifolds is not reversible. See [3].)

\section{The TORSION SUBGROUP}

In this section we shall describe the torsion subgroup of $H_{1}(M ; \mathbb{Z})$ in terms of the Seifert invariants of $M=M(g ; S)$.

Theorem 3.1. Let $M=M(g ; S)$ be an orientable 3-manifold which is Seifert fibred over an orientable base orbifold $B=T_{g}\left(\alpha_{1}, \ldots, \alpha_{r}\right)$. Then $H_{1}(M ; \mathbb{Z}) \cong$ $\mathbb{Z}^{2 g} \oplus\left(\bigoplus_{i \geq 0}\left(Z / \lambda_{i} Z\right)\right)$, where $\lambda_{i}$ is determined by $\left\{\alpha_{1}, \ldots, \alpha_{r}\right\}$ and is nonzero for all $i>0$, while $\left|\varepsilon_{S}\right| \Pi=\lambda_{0} \prod_{j \geq 1} \lambda_{j}$.

Proof. The fundamental group $\pi=\pi_{1}(M)$ has a presentation

$$
\left.\left\langle a_{1}, b_{1}, \ldots, b_{g}, q_{1}, \ldots, q_{r}, h\right| \prod\left[a_{i}, b_{i}\right] \prod q_{j}=1, q_{i}^{\alpha_{i}} h^{\beta_{i}}=1, h \text { central }\right\rangle .
$$

Hence $H_{1}(M ; \mathbb{Z}) \cong \mathbb{Z}^{2 g} \oplus \operatorname{Cok}(A)$, where the matrix $A$ is given by

$$
A=\left(\begin{array}{cccc}
0 & 1 & \ldots & 1 \\
\beta_{1} & \alpha_{1} & \ldots & 0 \\
\vdots & 0 & \ddots & \vdots \\
\beta_{r} & 0 & \ldots & \alpha_{r}
\end{array}\right)
$$

Let $E_{i}(A)$ be the ideal generated by the $(r+1-i) \times(r+1-i)$ subdeterminants of $A$, and let $\delta_{i}$ be the positive generator of $E_{i}(A)$. Then $\Delta_{0}=|\operatorname{det}(A)|=\left|\varepsilon_{S}\right| \Pi$. Since the elements of each row are relatively prime, $\Delta_{i}$ is the highest common factor of the $(r-i-1)$-fold products of distinct $\alpha_{j}$ s if $0<i<r$, and $\Delta_{i}=1$ if $i \geq \max \{r-1,1\}$. (In particular, if $r>2$, then $\Delta_{r-2}=\operatorname{hcf}\left(\alpha_{1}, \ldots, \alpha_{r}\right)$.) Thus $\Delta_{i}$ depends only on $\left\{\alpha_{1}, \ldots, \alpha_{r}\right\}$ and is nonzero for all $i>0$. If we set $\lambda_{i}=\Delta_{i} / \Delta_{i+1}$, for $i \geq 0$, then $\operatorname{Cok}(A) \cong \bigoplus_{i \geq 0}\left(Z / \lambda_{i} Z\right)$ by the Elementary Divisor Theorem. In particular, $\left|\varepsilon_{S}\right| \Pi=\lambda_{0} \prod_{j \geq 1} \lambda_{j}$.

Note that $T(M) \cong T(M(0 ; S))$ and the image of $h$ is in $T(M)$ if and only if $\varepsilon_{S} \neq 0$. 
Corollary 3.2. If $\Delta_{1}=1$, then $T(M)$ is cyclic, and $T(M)=0$ if and only if $\varepsilon_{S}=0$ or $\pm 1 / \Pi$. If $\Delta_{1}>1$, then $T(M) \neq 0$. Given $\left\{\alpha_{1}, \ldots, \alpha_{r}\right\}$ such that $\Delta_{1}>1$, there is at most one value of $|\varepsilon|$ for which the group $T(M)$ is a direct double.

Proof. As $\Delta_{1}=\prod_{i \geq 1} \lambda_{i}$ divides the order of $T(M)$, this group is nonzero unless $\Delta_{1}=1$. If $\varepsilon_{S}=0$, then $T(M) \cong \bigoplus_{i>1}\left(Z / \lambda_{i} Z\right)$, and so is a direct double if and only if $\lambda_{2 i-1}=\lambda_{2 i}$ for all $i>0$. If $\varepsilon_{S} \neq 0$, then $T(M) \cong \bigoplus_{i>0}\left(Z / \lambda_{i} Z\right)$, and so is a direct double if and only if $\lambda_{2 i}=\lambda_{2 i+1}$ for all $i \geq 0$. In particular, $\varepsilon_{S}=\left(\Delta_{1}\right)^{2} / \Pi \Delta_{2}$. Clearly these two systems of equations can both be satisfied only if $\lambda_{i}=1$ for all $i>0$ and $\lambda_{0}=0$ or 1 , in which case $T(M)=0$.

The elementary divisors $\lambda_{i}$ may be determined more explicitly by localization. If $p$ is a prime, an integer $\alpha$ has $p$-adic valuation $v$ if $\alpha=p^{v} q$, where $p$ does not divide $q$.

Corollary 3.3. Let $p$ be a prime and let $v_{i} \geq 0$ be the $p$-adic valuation of $\alpha_{i}$. Assume that the indexing is such that $v_{i} \geq v_{i+1}$ for all $i$. If $\varepsilon_{S}=0$ and $T(M)$ is a direct double, then $v_{2 j-1}=v_{2 j}$ for all $j \geq 1$.

Proof. The condition $v_{1}=v_{2}$ follows immediately from the fact that $p^{v_{2}} \varepsilon_{S}$ is an integer. The $p$-adic valuation of $\lambda_{j}$ is $v_{j+2}$, for all $j \geq 1$, and so $v_{2 j-1}=v_{2 j}$ for all $j \geq 2$ if $\bigoplus_{i \geq 1}\left(Z / \lambda_{i} Z\right)$ is a direct double.

If $M$ is Seifert fibred over a nonorientable base, then $T(M) \neq 0$ by Lemma 3.4 of [3]. Hence it follows from Theorem 3.1 that a Seifert fibred 3-manifold $M$ is a homology 3 -sphere if and only if $M=M(0 ; S)$ for some Seifert data $S$ with $\varepsilon_{S} \Pi= \pm 1$. In particular, $\operatorname{hcf}\left\{\alpha_{i}, \alpha_{j}\right\}=1$ for all $i<j \leq r$. Every homology 3 -sphere embeds as a TOP locally flat submanifold of $S^{4}[6]$.

Similarly, a Seifert fibred 3-manifold $M$ is a homology $S^{2} \times S^{1}$ if and only if $M=M(0 ; S)$ for some Seifert data $S$ with $\varepsilon_{S}=0$ and $\operatorname{hcf}\left\{\alpha_{i}, \alpha_{j}, \alpha_{k}\right\}=1$ for all $i<j<k \leq r$. Our main result settles the question of which Seifert fibred homology $S^{2} \times S^{1}$ s embed when all cone point orders are odd; the answer is not known in general. (If $M$ is a homology $S^{2} \times S^{1}$, there is an (essentially unique) degree-1 map $f: M \rightarrow S^{2} \times S^{1}$. If such a map $f$ induces an isomorphism with local coefficients $\Lambda=\mathbb{Z}[\mathbb{Z}]$, then $M$ embeds as a TOP locally flat submanifold of $S^{4}[10]$. However, this is not a necessary condition for embedding, and it can be shown that if $r>2$, then no such map with domain $M(0 ; S)$ is ever a $\Lambda$-homology equivalence.)

If $r \leq 2$ and $T(M)$ is a direct double, then $\varepsilon_{S} \Pi=0$ or \pm 1 , and so $M(0 ; S) \cong S^{3}$ or $S^{2} \times S^{1}$. More generally, if $r \leq 2$, then $M=M(g ; S)$ embeds in $S^{4}$ if and only if $T(M)=0$, in which case $M$ is a circle bundle over $T_{g}$ with Euler invariant 0 or \pm 1 .

\section{4. $\mathbb{H}^{2} \times \mathbb{E}^{1}$-MANIFOLDS WITH $g=0$}

If $S$ is skew-symmetric, then $\varepsilon_{S}=0$. If, moreover, the $\alpha_{i}$ s are all odd, a fibre sum construction based on embeddings of punctured lens spaces shows that $M(g ; S)$ embeds smoothly in $S^{4}$, for all $g \geq 0$. (See Lemma 3.1 of [3].) Here we shall show that when $\varepsilon_{S}=0$ and $g=0$, skew-symmetry is a necessary condition for $M(0 ; S)$ to embed in a homology 4 -sphere. 
If $M(g ; S)$ embeds with one complementary domain $X$ having a fixed-point free $S^{1}$-action, there is a direct, geometric argument as follows. The exceptional orbits with nontrivial isotropy subgroups have even codimension and are foliated by circles. Therefore they are tori (in the interior of $W$ ) and annuli with boundary components that are exceptional fibres of $M$. Consideration of relative orientations implies that the Seifert data of the boundary components of such annuli have the form $\{(\alpha, \beta),(\alpha,-\beta)\}$. However, if $X$ admits such an action, then $\chi(X)=0$.

Theorem 4.1. Let $M=M(g ; S)$ and let $\phi: \pi_{1}(M) \rightarrow \mathbb{Z}$ be an epimorphism such that $\phi(h) \neq 0$. If $b_{\phi}$ is neutral, then $S$ is skew-symmetric.

Proof. We note first that $\varepsilon_{S}=0$, since the image of $h$ in $H_{1}(M ; \mathbb{Z})$ has infinite order. Let $\sigma=|\phi(h)|$. Since $h$ is central, $\phi^{-1}(\sigma \mathbb{Z}) \cong \operatorname{Ker}(\phi) \times \mathbb{Z}$, and so the covering space associated to this subgroup is a product $F \times S^{1}$, where $F$ is a closed surface. Then $M$ fibres over $S^{1}$ with fibre $F$ and monodromy $\theta$ of order $\sigma$, and so the base orbifold $B$ is the quotient of $F$ by an effective action of $G=Z / \sigma Z$.

Let $\varphi: \pi^{o r b}(B)=\pi_{1}(M) /\langle h\rangle \rightarrow Z / \sigma Z$ be the epimorphism induced by $\phi$, and let $\tau \in G$ have image $\varphi(\tau)=[1]$. Then $F$ is the covering space associated to $\operatorname{Ker}(\varphi)$. The points $P$ with nontrivial isotropy subgroup $G_{P}$ lie above the cone points of $B$, and the representation of the isotropy subgroup $G_{P}=\left\langle\tau^{\sigma / \alpha_{i}}\right\rangle$ on the tangent space $T_{P}$ determines and is determined by the Seifert invariant $\left(\alpha_{i}, \beta_{i}\right)$ corresponding to the $i$ th cone point of $B$, since $\varphi\left(q_{i}\right)=-\left[\frac{\sigma}{\alpha}{ }_{i} \beta_{i}\right]$, for all $i \leq r$.

Since $M_{\phi} \cong F \times \mathbb{R}$ the Blanchfield pairing $b_{\phi}$ on $H_{1}\left(M_{\phi} ; \mathbb{Q}\right)$ reduces to the intersection pairing $I_{F}$ on the fibre $F$ together with the isometric action of $G=$ $\operatorname{Aut}(F / B) \cong Z / \sigma Z$. Let $s(n, k)=\left|\left\{i: \alpha_{i}=n, \beta_{i} \equiv k \bmod (n)\right\}\right|$ and $K(m)=\{k:$ $1 \leq k<m / 2,(k, m)=1\}$. Then the equivariant signatures of Atiyah, Bott and Singer are given by

$$
\operatorname{sign}\left(I_{F}, \tau^{\sigma / m}\right)=i \sum_{k \in K(m)}(s(m, m-k)-s(m, k)) \cot \left(\frac{\pi k}{m}\right),
$$

if $m>2$, by Theorem 6.27 of 1 .

If $b_{\phi}$ is neutral, its image in the Witt group $W(\mathbb{Q}(t), \mathbb{Q} \Lambda)$ is trivial. The equivariant signature $\operatorname{sign}\left(I_{F}, \tau^{\sigma / m}\right)$ is an invariant of the Witt class of $b_{\phi}$, for each $m \mid \sigma$. (See page 75 of [16].) Since $b_{\phi}$ is neutral these signatures are 0 . If $m>2$, the algebraic numbers $\left\{\cot \left(\frac{\pi k}{m}\right): k \in K(m)\right\}$ are linearly independent over $\mathbb{Q}[4$. Hence $s(m, m-k)=s(m, k)$ for all $k \in K(m)$ and $m>2$. Since we may modify each $\beta_{i}$ by multiples of $\alpha_{i}$, subject to $\varepsilon_{S}=0$, the Seifert data is skew-symmetric.

The converse is also true, but we shall only sketch the argument, as we do not need the result. Suppose that $S=\left\{\left(\alpha_{i}, \beta_{i}\right) \mid i \leq 2 s\right\}$ is skew-symmetric, and let $T=\left\{\left(\alpha_{2 j}, \beta_{2 j}\right) \mid j \leq s\right\}$. Then $M \cong N \#_{f}-N \#_{f} M(g ; \emptyset)$, where $N=M(0 ; T)$, and $\phi$ induces nonzero homomorphisms $\phi_{N}: \pi_{1}(N) \rightarrow Z$ and $\psi: \pi_{1}(M(g ; \emptyset)) \rightarrow Z$. Let $n(f)$ be an open tubular neighbourhood of a regular fibre of $N$, and let $N_{0}=$ $N \backslash n(f)$. Then $N \#_{f}-N=N_{0} \cup_{\partial}-N_{0}$. If $g=0$, then $\phi_{N}$ is an epimorphism and the diagonal copy of $H_{1}\left(N_{0} ; \mathbb{Q} \Lambda\right)$ in $H_{1}(M ; \mathbb{Q} \Lambda) \cong H_{1}\left(N_{0} ; \mathbb{Q} \Lambda\right) \oplus H_{1}\left(N_{0} ; \mathbb{Q} \Lambda\right)$ is a maximal self-annihilating submodule, so $b_{\phi}$ is neutral. In general, $\phi_{N}$ and $\psi$ need not be onto, and we must allow for infinite cyclic covering spaces with finitely many components. 
Lemma 4.2. Let $X$ be a compact 4-manifold with connected boundary $M$ such that $H_{1}(X, M ; \mathbb{Q})=0$. Suppose that $\Phi: \pi_{1}(X) \rightarrow \mathbb{Z}$ induces an epimorphism $\phi$ : $\pi_{1}(M) \rightarrow \mathbb{Z}$ such that $H_{1}(M ; \mathbb{Q}(t))=0$. Then $H_{2}(X, M ; \mathbb{Q}(t))=0 \Leftrightarrow \chi(X)=0$. If these conditions hold, then $b_{\phi}$ is neutral.

Proof. Since $H_{1}(M ; \mathbb{Q}(t))=H_{0}(M ; \mathbb{Q}(t))=0$ and $M_{\phi}$ is a connected open 3manifold, $H_{i}(M ; \mathbb{Q}(t))=0$, for all $i \geq 0$, and so $H_{i}(X ; \mathbb{Q}(t))=H_{i}(X, M ; \mathbb{Q}(t))$, for all $i>0$. Since $H_{1}(X, M ; \mathbb{Q})=0$ the module $H_{1}(X, M ; \mathbb{Q} \Lambda)$ is a torsion $\mathbb{Q} \Lambda$ module, so $H_{1}(X ; \mathbb{Q}(t))=0$ and $H^{1}(X, M ; \mathbb{Q}(t))=0$. Hence $H_{3}(X ; \mathbb{Q}(t))=0$ by Poincaré duality. Similarly, $H_{4}(X ; \mathbb{Q}(t))=H_{0}(X ; \mathbb{Q}(t))=0$. Since $\chi(X)$ may be calculated as the alternating sum of the dimensions of these $\mathbb{Q}(t)$-vector spaces, it follows that $\chi(X)=0$ if and only if $H_{2}(X, M ; \mathbb{Q}(t))=0$.

If these conditions hold, $H_{2}(W, M ; \mathbb{Q} \Lambda)$ is a $\mathbb{Q} \Lambda$-torsion module. Let $P$ be its image in $H_{1}(M ; \mathbb{Q} \Lambda)$. Then $P$ is its own annihilator with respect to $b_{\phi}$, and so $b_{\phi}$ is neutral. (See Theorem 2.3 of 9 .)

Corollary 4.3. If $M=M(g ; S)$ embeds in a homology 4-sphere $\Sigma=X \cup_{M} Y$, with $\chi(X)=0$ and $h$ having nonzero image in $H_{1}(X ; \mathbb{Q})$, then $S$ is skew-symmetric.

Proof. Let $\Phi: \pi_{1}(X) \rightarrow \mathbb{Z}$ be an epimorphism such that $\Phi(h) \neq 0$. The induced homomorphism $\phi: \pi_{1}(M) \rightarrow \mathbb{Z}$ is also an epimorphism, since $H_{1}(M ; \mathbb{Z})$ maps onto $H_{1}(X ; \mathbb{Z})$ and $H_{1}(M ; \mathbb{Q}(t))=0$. Thus $b_{\phi}$ is neutral by Lemma 4.2 , and so $S$ is skew-symmetric by Theorem 4.1.

In particular, this corollary applies when $g=0$.

Theorem 4.4. Let $M=M(0 ; S)$, where $\varepsilon=0$ and all cone point orders $\alpha_{i}$ of the base orbifold are odd. Then $M$ embeds if and only if the Seifert data is skewsymmetric.

Proof. Since $H_{2}(M ; \mathbb{Z}) \cong \mathbb{Z}$, we may assume that $H_{2}(X ; \mathbb{Z})=0$ and $H_{1}(X ; \mathbb{Z}) \cong$ $H_{1}(M ; \mathbb{Z})$. Hence $\chi(X)=0$ and $h$ has nonzero image in $H_{1}(X ; \mathbb{Q})$. Thus skewsymmetry is necessary by Corollary 4.3. It is also sufficient by Lemma 3.1 of [3].

If $\Sigma=X \cup_{M} Y$ with $H_{2}(X ; \mathbb{Z})=0$ and $\beta=\beta_{1}(M ; \mathbb{Q})$, the inclusions of $M$ and of $\vee^{\beta} S^{1}$ into $X$ induce isomorphisms on all the rational lower central series quotients of the fundamental groups [17. Hence these quotients are those of the free group $F(\beta)$. This is never true when $M$ is an $\mathbb{H}^{2} \times \mathbb{E}^{1}$-manifold with $\beta>1$, since the centre has nonzero image in $H_{1}(M ; \mathbb{Q})$. From the observations in $\S 2$ it follows that there is always a complementary component $X$ with $\chi(X)=0$ if $g \leq 1$. Does $h$ have nonzero image in $H_{1}(X ; \mathbb{Q})$ when $\chi(X)=0$ and $g=1$ ?

If $g>1$, the condition $\chi(X)=0$ holds for neither complementary region of $M(g ; \emptyset)=T_{g} \times S^{1}$, when embedded in $S^{4}$ as the boundary of a regular neighbourhood of an embedding of $T_{g}$. It remains possible that $b_{\phi}$ is neutral when $\Phi(h) \neq 0$ for some $\Phi$ as above. (This would follow from the argument of Theorem 2.3 in 9 if the torsion submodule of $H_{2}(X, M ; \mathbb{Q} \Lambda)$ maps onto the image of $H_{2}(X, M ; \mathbb{Q} \Lambda)$ in $H_{1}(M ; \mathbb{Q} \Lambda)$.)

When $r=2$ and $\varepsilon_{S}=0$, the Seifert data $S=\{(\alpha, \beta),(\alpha,-\beta)\}$ is skew-symmetric and $M=M(0 ; S) \cong S^{2} \times S^{1}$, with $S^{1}$-action given by $u .(w, z)=\left(u^{\alpha} w, u^{\beta} z\right)$ for $u, z \in S^{1}$ and $w \in \widehat{\mathbb{C}} \cong S^{2}$. If a regular fibre of $M$ bounds a locally flat disc in one complementary component of some embedding of $M$ in $S^{4}$, ambient surgery gives an embedding of $L \#-L$ in $S^{4}$, where $L=L(\alpha, \beta)$. This is only possible if 
$\alpha$ is odd [13. Thus it is not clear whether a fibre sum construction can be used to build up embeddings of other $\mathbb{H}^{2} \times \mathbb{E}^{1}$-manifolds with some exceptional fibres of even multiplicity.

If $S=\{(3,1),(5,-2),(15,1)\}$, then the $\alpha_{i}$ s are odd, $\varepsilon_{S}=0$ and $T(M(0 ; S))=0$, so $\ell_{M}$ is hyperbolic. Thus these conditions alone do not imply that $S$ must be skewsymmetric.

\section{Some REMARKS ON THE OTHER CASES}

We shall first make some comments on the results of 3 ] as they relate to $\mathbb{H}^{2} \times \mathbb{E}^{1}$ manifolds. Let $P_{k}=\#^{k} R P^{2}$ be a closed nonorientable surface with $\chi\left(P_{k}\right)=2-k$ and let $M=M(-k, S)$ be the Seifert fibred 3-manifold with base orbifold $B=$ $P_{k}\left(\alpha_{1}, \ldots, \alpha_{r}\right)$ and Seifert invariants $S=\left\{\left(\alpha_{1}, \beta_{1}\right), \ldots,\left(\alpha_{r}, \beta_{r}\right)\right\}$, for $k \geq 1$. In this case $T(M)$ is again largely determined by the set $\left\{\alpha_{1}, \ldots, \alpha_{r}\right\}$, but $\varepsilon_{S}=$ $-\sum_{i=1}^{i=r}\left(\beta_{i} / \alpha_{i}\right)$ is not constrained at all by the condition that $T(M)$ be a direct double.

We shall assume that all cone point orders $\alpha_{i}$ are odd. (Then $M$ is an $\mathbb{H}^{2} \times \mathbb{E}^{1}$ manifold or a $\widetilde{\mathbb{S L}}$-manifold unless $k+r \leq 2$.) Since $\varepsilon_{S}$ is a rational number with odd denominator, it has a well-defined image in $Z / 2^{s} Z$, for any $s \geq 0$. In particular, $\varepsilon_{S} \equiv \sum \beta_{i} \bmod (2)$ and $\varepsilon_{S} \equiv-\sum \alpha_{i} \beta_{i} \bmod (4)$, since $\alpha_{i} \equiv 1 \bmod (2)$ and $\alpha_{i} \equiv \alpha_{i}^{-1} \bmod (4)$ if $\alpha_{i}$ is odd. Thus the invariants $c$ and $\eta$ used in Lemma 3.4 and Theorem 3.7 of [3] are just the images of $-\varepsilon_{S}$ in $Z / 2 Z$ and $Z / 4 Z$ (respectively). Hence $\varepsilon_{S} \equiv 2 k \bmod (4)$ if $\ell_{M}$ is hyperbolic by Theorem 3.7 of 3 .

If $\varepsilon_{S}=0$, then $T(M) \cong(Z / 2 Z)^{2} \oplus \bigoplus_{i>1} Z / \alpha_{i} Z$ by Theorem 3.7 of [3]. Therefore if $T(M)$ is a direct double, the numbers $\#\left\{i: v_{p}\left(\alpha_{i}\right)=j\right\}$ are even for all odd primes $p$ and exponents $j \geq 1$. If, moreover, $r=3$, then it follows from Lemma 3.6 of $[3$ ] that $\ell_{M}$ is hyperbolic. Must $S$ be skew-symmetric if $\varepsilon_{S}=0$ and $M(-k, S)$ embeds in $S^{4}$ ? The first cases to test are perhaps those with base $P_{2}(p, q, p q)$, where $p$ and $q$ are distinct odd primes. (When $k=2$, one complementary domain, $X$ say, has $H_{1}(M ; \mathbb{Q}) \cong H_{1}(X ; \mathbb{Q})$ and $\chi(X)=0$. However, the argument of Theorem 4.1 does not appear to apply usefully here, as we must first pass to the 2 -fold cover of $M$ induced by the orientation cover of the base $B$ before continuing to an infinite cyclic cover homeomorphic to $F \times \mathbb{R}$. There is no obvious reason that the Blanchfield pairing associated to the latter cover should be neutral.)

The situation is less clear when $\varepsilon_{S} \neq 0$. If $\varepsilon_{S} \Pi=1$, then $M(g ; S)$ embeds in $S^{4}$ for all $g \geq 0$, since $M(0 ; S)$ is a homology 3 -sphere. It is easy to find such examples for any $r \geq 1$. Thus there is no reason to expect parity constraints for embedding such manifolds. (However, the question of which Seifert fibred homology 3-spheres embed smoothly is still open. See Problem 4.2 of [14.)

\section{REFERENCES}

1. M. F. Atiyah and R. Bott, A Lefschetz fixed point theorem for elliptic complexes. II. Applications, Ann. Math. 88 (1968), 451-491. MR0232406 (38:731)

2. R. Budney, Embedding of 3-manifolds in $S^{4}$ from the point of view of the 11-tetrahedron census, arXiv: 0810.2346v1 [math.GT].

3. J. S. Crisp and J. A. Hillman, Embedding Seifert fibred and Sol ${ }^{3}$-manifolds in 4-space, Proc. London Math. Soc. 76 (1998), 685-710. MR1620508 (99g:57027)

4. A. L. Edmonds and J. H. Ewing, Remarks on the cobordism group of surface diffeomorphisms, Math. Ann. 259 (1982), 497-504. MR660044 (83m:57026) 
5. D. B. A. Epstein, Embedding punctured manifolds, Proc. Amer. Math. Soc. 16 (1965), 175176. MR0208606 (34:8415)

6. M. H. Freedman, The topology of four-dimensional manifolds, J. Diff. Geom. 17 (1982), 357453. MR679066 (84b:57006)

7. P. Gilmer and C. Livingston, On embedding 3-manifolds in 4-space, Topology 22 (1983), 241-252. MR710099 (85b:57035)

8. W. Hantzsche, Einlagerung von Mannigfaltigkeiten in euklidische Räume, Math. Z. 43 (1937), 38-58. MR 1545714

9. J. A. Hillman, Algebraic Invariants of Links, World Scientific Publishing Co. Pte Ltd. (2002). MR.1932169 (2003k:57014)

10. J. A. Hillman, Embedding homology equivalent 3-manifolds in 4-space, Math. Z. 223 (1996), 473-481. MR:1417856 (97h:57044)

11. M. Jankins and W. D. Neumann, Lectures on Seifert Manifolds, Brandeis Lecture Notes 2, Brandeis University (1983). MR741334 (85j:57015)

12. A. Kawauchi, On quadratic forms of 3-manifolds, Invent. Math. 43 (1977), 177-198. MR.0488074 (58:7645)

13. A. Kawauchi and S. Kojima, Algebraic classification of linking pairings on 3-manifolds, Math. Ann. 253 (1980), 29-42. MR594531 (82b:57007)

14. R. C. Kirby, Problems in low-dimensional topology, in Geometric Topology (edited by W. H. Kazez), Part 2, Amer. Math. Soc. and International Press (1997), 35-473. MR.1470751

15. R. A. Litherland, Cobordism of satellite knots, in Four Manifold Theory, Contemp. Math., 35, Amer. Math. Soc. (1984), 327-362. MR780587 (86k:57003)

16. W. D. Neumann, Equivariant Witt Rings, Bonner Mathematische Schriften 100, Universität Bonn (1977). MR 494248(80a:57010)

17. J. R. Stallings, Homology and central series of groups, J. Algebra 2 (1965), 170-181. MR0175956 (31:232)

School of Mathematics and Statistics, University of Sydney, Sydney, NSW 2006, Australia

E-mail address: jonh@maths.usyd.edu.au 